NACIONAL

\title{
Las personas con discapacidad en el proceso constituyente chileno
}

\author{
Persons with disabilities in the Chilean constitution-making process
}

\section{Pablo Marshall (iD Viviana Ponce de León (iD y Eduardo Marchant (iD Universidad Austral de Chile}

\begin{abstract}
RESUMEN Este trabajo aborda las condiciones para una participación política efectiva de las personas con discapacidad en el proceso constituyente en curso en Chile. Para ello, comienza introduciendo el marco conceptual que identifica las potencialidades y dificultades que dicha participación plantea. Luego, se examina el marco normativo que, conforme al derecho internacional de los derechos humanos, informa la participación política y electoral de personas con discapacidad en general. A la luz de este marco normativo, se describen los requisitos de accesibilidad general que deberían implementarse para garantizar esa participación en el proceso constituyente. Por último, se describe y analiza el mecanismo en discusión para canalizar las demandas sociales por un órgano constituyente inclusivo.
\end{abstract}

PALABRAS CLAVE Discapacidad, proceso constituyente, participación, inclusión, accesibilidad.

ABSTRACT This paper addresses the conditions for effective political participation of persons with disabilities in Chile's current constitution-making process. To that effect, it starts by introducing the conceptual framework that identifies the potentialities and difficulties that such participation entails. Then, it examines the normative framework that, under international human rights law, informs the political and electoral participation of persons with disabilities. Considering this normative framework, it describes the general accessibility requirements that should be implemented to guarantee participation in the constitution-making process. Finally, it describes and analyzes the currently discussed mechanism to channel the social demands for an inclusive constitution-making body.

KEYWORDS Disability, constitution-making process, participation, accessibility. 


\section{Introducción}

El objetivo del presente trabajo es analizar uno de los elementos necesarios para que un proceso constituyente tenga el potencial de reflexionar sobre la posición que las personas con discapacidad ocupan en la sociedad: garantizar su participación política efectiva en el proceso constituyente. Queremos dar cuenta de las potencialidades y dificultades que dicho camino enfrenta, considerando tanto las medidas que han sido adoptadas para permitir esa participación, como la literatura que ha abordado el fenómeno de la participación política de las personas con discapacidad. Para ello, en primer lugar, se introduce un marco conceptual que identifica el reto de la transformación constitucional que vaya en beneficio de las personas con discapacidad; en segundo lugar, se identifica un marco normativo en el que, conforme al derecho internacional de los derechos humanos, la participación de las personas con discapacidad debe desarrollarse; en tercer lugar, se describen requisitos de accesibilidad general que deberían implementarse para la participación electoral y plebiscitaria en el contexto del proceso constituyente; en cuarto lugar, se describe y analiza el mecanismo que ha sido incorporado al proceso constituyente en curso para la participación de las personas con discapacidad en el órgano constituyente.

\section{¿Es el proceso constituyente una oportunidad para las personas con discapacidad?}

\section{La sociedad ableista}

Las personas con discapacidad han sido tradicionalmente marginadas de la participación y la toma de decisiones políticas. Como consecuencia, es usual que vean que sus intereses son desplazados de la discusión y de las decisiones que se adoptan, lo que resulta en especial problemático desde un punto de vista democrático cuando se trata de las decisiones que les afectan. Esto se deriva de un gran número de razones y se expresa en varias formas. Por ejemplo, se ha considerado que las personas con discapacidad intelectual no tienen la capacidad o la independencia para participar en la toma de decisiones políticas (Marshall y Abollado, 2019). Otro ejemplo es el de la falta de mecanismos de votación que permitan sufragar a personas que no se pueden desplazar a los locales de votación (Bundy, 2003: 217-240; Schur, Ameri y Adya, 2017: 1.374-1.39o). Una idea general que subyace a todas esas razones y a las formas en que se expresan consiste en sostener que la sociedad está organizada de una forma especialmente hostil para las personas con discapacidad o, dicho de otro modo, que la sociedad en su totalidad está sesgada de una manera que las perjudica en forma sistemática. Si queremos traducir la operación de dichos sesgos u hostilidad a una idea más cercana al lenguaje de los derechos humanos de las personas con discapacidad, siguiendo el modelo social de la discapacidad, podemos decir que ellos funcionan a 
través de un conjunto de dispositivos o estructuras que se traducen en barreras para el ejercicio de derechos (Degener, 2017: 41-59).

Tal como el machismo es criticado por los estudios de género por privilegiar un modelo masculino de sociedad sobre uno que incluya y empodere a las mujeres, los estudios culturales de la discapacidad han acuñado su propio término: el ableismo o capacitismo (Goodley, 2014). El ableismo se deriva del adjetivo inglés able, que quiere decir hábil o apto, y corresponde, por tanto, a la disposición de grupos y estructuras sociales a valorar y promover ciertas habilidades por sobre otras - por ejemplo, las relacionadas con la productividad y la competencia sobre la compasión y la amabilidad - asumiendo que la falta de aquellas habilidades consideradas como esenciales define a una persona como menos valiosa. Aunque puede operar contra las mujeres y otras minorías, el ableismo contra las personas con discapacidad es el caso más tratado de este fenómeno. Ciertas habilidades de las personas son normalizadas en contra de las personas que tienen una deficiencia médicamente diagnosticada, lo que establece una jerarquía entre las personas con y sin discapacidades (Wolbring, 2007: 252-258). Esta jerarquía tiende a atribuir el poder, y las ventajas y beneficios que son distribuidos por la sociedad, a los individuos sin discapacidad, y a privar de ello $a$ priori, de manera discriminatoria, a las personas con discapacidad. Una sociedad ableista, por tanto, es una que discrimina y está prejuiciada, no siempre de manera consciente y deliberada, contra las personas con discapacidad. Ello implica, por ejemplo, que el diseño del acceso a los espacios públicos no toma en consideración las dificultades que las personas con discapacidad experimentan, lo que acarrea que no puedan aparecer, o al menos no de igual manera que los demás, en dichos espacios. Lo anterior bien podría deberse a que la sociedad tiende a invisibilizar sus intereses en la medida que no son escuchadas en la esfera pública y no aparecen en un espacio público. Debido a que sus intereses son invisibles, la sociedad es diseñada de una forma en que no las toma en consideración. Para revertir la exclusión de la que son objeto, es importante tomar conciencia, primero, de que vivimos en una sociedad ableista, como en la última década se ha tomado conciencia de que vivimos en una sociedad machista. Luego, debemos adoptar medidas tanto para visibilizar como para empoderar a las personas con discapacidad.

Si bien el concepto de ableismo es producto de una determinada concepción de la discapacidad, ligada a los estudios culturales de orden posmoderno acerca de la discapacidad, es un término útil para describir que el conjunto de formas específicas de exclusión y barreras para el ejercicio de derechos de las personas con discapacidad tienen un origen estructural y son producto de una construcción social que es posible de transformar. Desde la perspectiva del derecho, los derechos reconocidos a las demás personas, por lo general, también les son reconocidos a las personas con discapacidad (Raub y otros, 2016: 203-240). Sin embargo, el ejercicio de dichos derechos en igualdad de condiciones es impedido, en la medida que su implementación 
ha sido llevada a cabo en una sociedad ableista. Las personas con discapacidad enfrentan barreras de la más diversa índole: actitudinales, físicas, jurídicas, económicas, sociales y de comunicación, que un proceso constituyente tiene la potencialidad de ayudar a remediar.

\section{Una constitución para las personas con discapacidad}

La Convención de Derechos de las Personas con Discapacidad (CDPD) ha sido fundamental para visibilizar las barreras que las personas con discapacidad enfrentan para ejercer sus derechos. En el lenguaje de la CDPD, la discapacidad es concebida como un conjunto de barreras para el ejercicio de derechos que se manifiestan cuando las personas con discapacidad interactúan con la sociedad. El problema, en esta línea, no está en las personas, sino en la sociedad opresora. Es por eso que la CDPD no establece nuevos derechos, sino que se enfoca en cómo los derechos civiles, políticos y sociales tradicionales deben entenderse para evitar las barreras en su ejercicio. Por ejemplo, el derecho a reunirse en el espacio público es más difícil y muchas veces imposible de ejercer para una persona que debe movilizarse en silla de ruedas; el ejercicio del derecho a sufragio está prohibido por el derecho para muchas personas con discapacidad mental (artículo 16 numeral primero de la Constitución) y los locales de votación son muchas veces inaccesibles para personas con movilidad reducida; el ejercicio del derecho a la libertad de expresión es más difícil y muchas veces imposible para personas sordas, que requieren un destinatario que entienda la lengua de señas para ser comprendidas.

Éstos son sólo algunos ejemplos de las dificultades que experimentan las personas con discapacidad en el ejercicio de sus derechos de participación. Si bien nuestra sociedad no es insensible a dichas barreras y algunos esfuerzos han sido desplegados para eliminarlas - por ejemplo, estableciendo mecanismos alternativos para sufragar-, esos esfuerzos son, como veremos, todavía insuficientes.

Un estudio publicado en 2016 muestra el nivel de recepción de normas constitucionales específicamente dirigidas a garantizar los derechos de las personas con discapacidad en un universo de 193 jurisdicciones. Los derechos específicos que más comúnmente se encuentran consagrados de manera constitucional en el derecho comparado dicen relación con derechos de contenido social: el derecho a la educación (28\%), a la salud (26\%) y al trabajo y la no discriminación en el empleo (18\%). Asimismo, hay un número relevante de constituciones que establecen una regulación específica del derecho a la igualdad y no discriminación $(24 \%)^{1}$ y de los derechos

1. El proyecto de reforma constitucional con el que concluyó el proceso constituyente de la presidenta Michelle Bachelet (Boletín 11.617-07) consagró a la discapacidad como una categoría de la cláusula antidiscriminación del artículo 19, numeral cuarto. 
de participación política (22\%) en relación con las personas con discapacidad. Por último, en un número mucho más limitado se ubica la garantía de la libertad de movimiento y expresión ( $9 \%$ ) y el derecho de asociación (7\%) (Raub y otros, 2016: 235-236). La garantía de derechos constitucionales que hagan específica mención a las personas con discapacidad en su regulación no es una solución definitiva al problema de las barreras de acceso que afectan a la población con discapacidad. Es, sin embargo, un primer paso. Se requiere también una legislación complementaria, una actividad de litigación judicial y una movilización social acorde, para garantizar que los derechos consagrados constitucionalmente se cumplan en la práctica. Las disposiciones constitucionales no sólo tienen un efecto regulativo que puede ser importante para la garantía de los derechos de las personas con discapacidad, por la vía legislativa y judicial, sino que también tienen un efecto simbólico importante. Ese efecto puede contribuir a promover no sólo el respeto de los derechos de las personas con discapacidad, sino también una visión de la sociedad en su conjunto en que las preocupaciones de las personas con discapacidad no sean invisibilizadas.

La consagración de regulaciones específicas para las personas con discapacidad -en relación con los derechos fundamentales tradicionalmente consagrados para quienes no tienen una discapacidad - no agota el potencial contenido de una nueva constitución al servicio de la eliminación de las barreras que experimentan en el ejercicio de sus derechos. Hay dos caminos adicionales, inéditos en el diseño constitucional comparado, que pueden adoptarse a la hora de la redacción de las nuevas disposiciones constitucionales. En primer lugar, está la idea de un diseño institucional inclusivo, eso es, pensar no sólo los derechos, sino también la composición de los órganos y la consagración de atribuciones a dichos órganos de una forma que los haga accesibles a las personas con discapacidad. En segundo lugar, se encuentra la posibilidad de incluir cláusulas específicamente dirigidas a la protección de las personas con discapacidad, por ejemplo, incluyendo la obligación de todos los órganos del Estado de resguardar el principio de accesibilidad o el principio de acceso universal en su caso, y el derecho a los ajustes razonables para las personas con discapacidad. Es el caso, por ejemplo, de los artículos 70 a 72 de la Constitución boliviana, que garantizan derechos específicos de las personas con discapacidad: la prohibición de la discriminación, maltrato y violencia, y el compromiso del Estado con acciones positivas, entre otros. ${ }^{2}$ Una cláusula con dicho contenido también podría establecerse en la regulación del derecho a la igualdad y no discriminación. Estas alternativas son complementarias y podrían fortalecer la protección de los derechos de las personas con discapacidad.

2. Otras constituciones que establecen una regulación especial para las personas con discapacidad son, por ejemplo, las de Ecuador (artículos 47 a 49), Zimbabue (artículo 22), Kenia (artículo 54) y Uganda (artículo 35). 


\section{La contribución que las personas con discapacidad pueden hacer al proceso constituyente}

Es sin duda importante que las personas con discapacidad puedan participar en la creación de una nueva constitución. Ello viene dado por el necesario respeto a su derecho a autodeterminarse individualmente y a formar parte, mediante el ejercicio de un conjunto de derechos, de la autodeterminación colectiva. El derecho a participar ha quedado establecido tanto en la CDPD como en las legislaciones nacionales. Desde esta perspectiva, las personas con discapacidad tienen derecho a participar y dicho derecho debe ser respetado por consideraciones de principio.

Sin embargo, su participación no sólo puede ser defendida por razones de principio. También puede ser bienvenida por razones de justicia epistémica, esto es, desde el punto de vista de la específica contribución que con su conocimiento único sólo ellas pueden entregar (Tremain, 2016), en este caso, al proceso de discusión de una nueva constitución. En esta línea, sólo las personas con discapacidad, a través de los conocimientos aprendidos por sus propias experiencias de vida, pueden perfilar con detalle las medidas que deben ser adoptadas para concebir una constitución que permita la construcción de una sociedad no ableista, en la que tengan igual oportunidad de desarrollar sus planes de vida individuales y participar en proyectos colectivos. Esto supone, por un lado, reconocer la autoridad epistémica de las personas con discapacidad en los asuntos que implican comprender sus propias experiencias. Por otro lado, exige un esfuerzo de humildad de parte de las personas sin discapacidad, para reconocer que es muy difícil o incluso imposible entender qué es lo que significa ponerse en el lugar del otro, cuando ese otro ha sido, por largo tiempo, alguien invisible tanto en el espacio público como en las representaciones culturales de la sociedad.

Con todo, la contribución que las personas con discapacidad pueden hacer, obviamente, no proviene sólo de su experiencia personal. Por un lado, esta contribución es mucho más rica y de seguro incluirá puntos de vista, razones y argumentos novedosos, críticos y desafiantes para las formas en que las personas sin discapacidad han pensado la sociedad. Por otro lado, incluso contra todas las barreras que hacen su organización improbable y difícil, las personas con discapacidad se han organizado en redes de colaboración y apoyo que les permitirán articular sus demandas de una forma colectiva. Ejemplo de esta capacidad de la colectividad es la carta que en junio de 2020 un grupo de dirigentes de organizaciones de y para las personas con discapacidad enviaron al Congreso Nacional solicitando el posicionamiento de la discapacidad como un tema político y la garantía del derecho de participación en el proceso constituyente, ${ }^{3}$ además de reivindicar la contribución de su perspectiva en la

3. Pablo Selles, «Carta de organizaciones sociales», El Mostrador, 31 de julio de 2020, disponible en https://bit.ly/37f $Z_{7} \mathrm{G}$. 
creación de un marco inclusivo para todos los ciudadanos, esto en el debate sobre la regulación del proceso constituyente.

La contribución de las personas con discapacidad no sólo puede impactar en la eliminación o la mitigación del ableismo de nuestra sociedad y la consecuente eliminación de barreras que las afectan. Su contribución puede tener mayores alcances. Contar con una activa participación de personas con discapacidad en la discusión constituyente contribuirá a la elaboración de un mejor arreglo social para todos los que aún no tenemos ninguna discapacidad, en la medida que puede contribuir a visibilizar, como ha defendido - entre otros - Johnatan Herring, la vulnerabilidad inherente de cualquier ser humano y el cuidado que todos requerimos en algún momento de nuestras vidas adultas (Herring, 2013). Para entender esto, debemos recordar que la discapacidad es un fenómeno mucho más extendido que lo que se piensa. Según el último estudio nacional sobre la discapacidad, cerca de 2,9 millones de personas, aproximadamente el $16,7 \%$ de la población nacional, posee algún tipo de discapacidad, ${ }^{4}$ y la prevalencia de la discapacidad sólo puede tender a extenderse. El envejecimiento de la población va a hacer que las discapacidades relacionadas con la edad se multipliquen y que ambientes y servicios más accesibles se transformen en una necesidad urgente. Esto puede confirmar la idea de que todos somos potenciales personas con discapacidad, pero, de manera más radical, puede contribuir a que entendamos que todos somos dependientes unos de otros. Las ideas de vulnerabilidad, interdependencia y solidaridad pueden constituir un aporte propio de la tradición de la lucha de las personas con discapacidad al proceso constituyente.

\section{Las exigencias del derecho internacional y su puesta en práctica}

Los ejes normativos en torno a la participación de las personas con discapacidad

El derecho de participación se encuentra garantizado a todas las personas en una serie de instrumentos internacionales de derechos humanos. Así puede verse en la Declaración Universal de Derechos Humanos (artículo 21), el Pacto Internacional de Derechos Civiles y Políticos (artículo 25) y la Convención Americana de Derechos Humanos (artículo 23.1), entre otros. No obstante, a efectos de la participación política de personas con discapacidad, el instrumento más relevante en esta materia es la ya mencionada CDPD. En ella se establecen una serie de principios generales, como el respeto de la dignidad inherente y la autonomía individual de las personas con discapacidad, la no discriminación, la igualdad de oportunidades y la accesibilidad, entre otros. Todos ellos son importantes de considerar en la redacción de una

4. «Segundo Estudio Nacional de la Discapacidad», Servicio Nacional de la Discapacidad, 2015, disponible en https://bit.ly/3mhyNaf. 
nueva constitución, en la medida que establecen obligaciones internacionales para el Estado. Más específicamente, en lo que respecta a la inclusión de personas con discapacidad en el marco del proceso constituyente, hay al menos dos artículos de la CDPD que aportan orientaciones relevantes. ${ }^{5}$

El primero es el artículo 4 párrafo 3, que impone a los Estados parte el deber de celebrar consultas y colaborar activamente con personas con discapacidad para la elaboración y aplicación de legislación y políticas para hacer efectiva la CDPD, además de en otros procesos de adopción de decisiones sobre cuestiones relacionadas con las personas con discapacidad. Esta disposición hace eco de la consigna acuñada por los movimientos y agrupaciones de personas con discapacidad: «Nada sobre nosotros sin nosotros». Según lo ha expresado el Comité sobre los Derechos de las Personas con Discapacidad en su Observación General 7, las cuestiones relacionadas con las personas con discapacidad podrían, justamente, «guardar relación con el derecho constitucional, los derechos electorales, el acceso a la justicia, el nombramiento de las autoridades administrativas a cargo de las políticas en materia de discapacidad o las políticas públicas en los ámbitos de la educación, la salud, el trabajo y el empleo» (párrafo 20). El Comité también ha puntualizado que «los Estados parte deberían considerar las consultas y la integración de las personas con discapacidad como medida obligatoria antes de aprobar leyes, reglamentos y políticas, ya sean de carácter general o relativos a la discapacidad» (párrafo 15).

Es así como la consulta se erige en una etapa ineludible en el proceso constituyente, a la luz de este deber del Estado, toda vez que en él surgirán diversas cuestiones que afectarán directa o indirectamente a las personas con discapacidad. Dichas consultas deberían tener lugar en las fases iniciales de los procesos de adopción de decisiones y contribuir en sus resultados finales. En ellas se deberían considerar a todas las personas con discapacidad, incluyendo a las personas con discapacidad sicosocial e intelectual, así como también a las organizaciones de personas con discapacidad que las agrupan y representan sus intereses.

En cuanto a su valor, el Comité ha precisado que las opiniones recabadas en las consultas deben ser debidamente consideradas. En consecuencia, los Estados parte deben garantizar que se escuche a las personas con discapacidad no como una mera formalidad o gesto simbólico, sino teniendo en cuenta las observaciones y demandas

5. Con todo, el énfasis en los dos artículos que se analizarán no implica que no existan otras disposiciones de la CDPD cuyo contenido normativo impacte en el ejercicio de derechos políticos por parte de las personas con discapacidad. Sin ánimo de exhaustividad, es posible vincular las exigencias de participación política con el derecho a la igualdad y no discriminación (artículo 5), con la obligación de proporcionar accesibilidad (artículo 9), con el derecho al igual reconocimiento como persona ante la ley (artículo 12) y con el derecho a vivir en forma independiente y a ser incluido en la comunidad (artículo 19). 
que hagan, de manera que éstas se reflejen en las decisiones que se adopten (párrafo 48). Igualmente, deben informar a las organizaciones de personas con discapacidad acerca de los resultados de esos procesos y proporcionarles una explicación clara acerca del modo en que se tuvieron en cuenta sus opiniones (párrafo 23).

El segundo artículo relevante es el artículo 29 de la CDPD, referido a la participación política y pública. Establece el deber de garantizar a las personas con discapacidad los derechos políticos y la posibilidad de gozar de ellos en igualdad de condiciones que las demás. ${ }^{6}$ Para ello, se debe asegurar que puedan participar de manera plena y efectiva en la vida política y pública, en forma directa o a través de representantes libremente elegidos, incluidos su derecho y posibilidad de votar y ser elegidas. Asimismo, prevé la obligación de promover en forma activa un entorno en el que las personas con discapacidad puedan participar plena y efectivamente en la dirección de los asuntos públicos, ${ }^{7}$ sin discriminación y en igualdad de condiciones con las demás. ${ }^{8}$ Que la participación de las personas con discapacidad sea plena supone su presencia regular en reuniones de alto nivel y en negociaciones. Que sea efectiva, por su parte, implica que sus contribuciones deben reflejarse en la decisión final que se adopte (Birtha, 2013: 115-138).

Aunque el Comité ha proporcionado una serie de directrices para satisfacer estas exigencias, ${ }^{9}$ existen todavía brechas considerables en su implementación (Birtha,

6. Desde la perspectiva del derecho al igual reconocimiento ante la ley (artículo 12 de la CDPD), el Comité ha resaltado que el reconocimiento de la capacidad jurídica de las personas con discapacidad en todos los aspectos de la vida incluye la participación en la vida política y pública. Además, prosigue el Comité, la capacidad para tomar decisiones no puede operar como criterio de justificación para excluirlas del ejercicio de los derechos políticos (Observación General 1, párrafo 48).

7. A través de la participación plena y efectiva en la dirección en los asuntos públicos, las personas con discapacidad concretizan, además, su derecho a ser incluidos en la comunidad (artículo 19 de la CDPD). En este sentido, el Comité ha explicitado la relación entre los derechos consagrados en los artículos 19 y 29 del CDPD, indicando que "para influir y participar en las decisiones que repercuten en el desarrollo de su comunidad, todas las personas con discapacidad deben disfrutar y ejercer sus derechos de participación en la vida política y pública (artículo 29) de manera individual o a través de sus organizaciones» (Observación General 5, párrafo 93).

8. Con relación al derecho de igualdad y no discriminación (artículo 5 de la CDPD), el Comité ha enumerado una serie de medidas que apuntan a evitar la discriminación en el goce y ejercicio del derecho a la participación política y pública de las personas con discapacidad. Entre otras medidas, cabe resaltar: i) la derogación las restricciones legales que les impiden el ejercicio del derecho de voto o de presentarse como candidatos en elecciones populares; ii) la garantía de que todas las etapas del proceso electoral les sean accesibles; y iii) la realización de ajustes razonables y la adopción de medidas de apoyo para las personas con discapacidad que así lo requieran (Observación General 6, párrafo 70).

9. Cabe resaltar que en el ámbito del sistema de protección universal de derechos humanos, no sólo el Comité se ha preocupado de abordar la participación política de las personas con discapacidad. En efecto, en los últimos años, tanto la Oficina del Alto Comisionado de las Naciones Unidas para los Derechos Humanos como la Relatoría Especial sobre los derechos de las personas con discapacidad han presenta- 
2013: 115; Lang y otros, 2011: 206-220; Levesque y Langford, 2016: 62-102; Sherlaw y Hudebine, 2015: 9-21; Waldschmit y otros, 2017: 177-195). Entre los criterios que podrían contribuir a reducir estas brechas se encuentran la transparencia, la participación de personas con discapacidad desde las etapas iniciales de los procesos de toma de decisiones, la colaboración amplia de todos los actores relevantes, el despliegue de campañas educativas para generar conciencia, la evaluación continua y la influencia directa en las decisiones mediante la provisión de información basada en evidencia (Birtha, 2013: 133). En complemento a estas ideas, el Comité reconoce el derecho a la participación plena y efectiva como un derecho civil y político de las personas con discapacidad y, correlativamente, como una obligación de cumplimiento inmediato para los Estados parte, sin dilación y sin sujeción a ninguna forma de restricción presupuestaria. De igual manera, ha señalado que este derecho no se limita a la sola adopción de decisiones públicas relativas a la CDPD, sino que se extiende también a la implementación y seguimiento. En este sentido, «la participación plena y efectiva debería entenderse como un proceso y no como un acontecimiento puntual aislado» (Observación General 7, párrafo 28).

\section{El rol de las organizaciones de personas con discapacidad}

Tanto el deber de consulta como el derecho a participar en la vida política y pública se reconocen, sin duda, respecto de las personas con discapacidad consideradas en forma individual. Sin embargo, ambos se hacen extensivos también a las organizaciones que son dirigidas, administradas y gobernadas por y cuyos miembros son mayoritariamente personas con discapacidad. Dichas organizaciones son creadas con el fin de actuar, expresar, promover, perseguir o defender los derechos de las personas con discapacidad.

La participación significativa de estas organizaciones suele enfrentar una serie de desafíos. Entre ellos cabe destacar, primero, la falta de recursos y capacidad técnica para conducir investigación que pueda informar la implementación de la CDPD; segundo, la ausencia de una voz uniforme y una participación equilibrada de las organizaciones (Kamundia, 2014: 185-205; Birtha, 2013: 133-136; Lang y otros, 2011: 213). Para abordar estas dificultades, el Comité ha establecido que los Estados parte deben proporcionar el apoyo y financiamiento necesario para garantizar la participación de

\footnotetext{
do al Consejo de Derechos Humanos sendos informes temáticos sobre la participación de las personas con discapacidad en la vida política y pública. Véase «Estudio temático preparado por la Oficina del Alto Comisionado de las Naciones Unidas para los Derechos Humanos sobre la participación de las personas con discapacidad en la vida política y pública», Consejo de Derechos Humanos, A/HRC/19/36, 30 de noviembre de 2011, disponible en https://bit.ly/3804M9K; e «Informe de la relatora especial sobre los derechos de las personas con discapacidad», Consejo de Derechos Humanos, A/HRC/31/62, 12 de enero de 2016, disponible en https://undocs.org/A/HRC/31/62.
} 
los representantes de todas las personas con discapacidad en los procesos de consulta, en todos los niveles de adopción de decisiones y en los procesos de aplicación y seguimiento de la CDPD (Observación General 6, párrafos 7, 39, 45, 46, 60-64 y 94). Por otra parte, es importante asegurar la participación de organizaciones que reflejen la diversidad dentro de la discapacidad, así como a una variedad de segmentos sociales y grupos etarios. Especial consideración ha de darse a la participación significativa de personas con discapacidad que experimentan formas de discriminación múltiples e interseccionales, como mujeres, niñas y niños, indígenas minorías étnicas, refugiados y personas que viven en zonas rurales o remotas, entre otros (Rattray y Lako, 2018; Toritsyn y Kabir, 2013). Del mismo modo, debe considerarse un rango amplio de formas organizacionales (Rattray y Lako, 2018: 72).

En términos del impacto de la intervención de las organizaciones de personas con discapacidad, una estrategia relativamente exitosa a nivel comparado consiste en conformar grupos de trabajo unitarios entre ellas (Löve, Traustadóttir y Rice, 2019: 1-15; Sherlaw y Hudebine, 2015: 15-16). Estos grupos de trabajo se centran exclusivamente en determinados asuntos fundamentales en los que las posiciones de las personas con discapacidad están alineadas y presentan un frente unido. Por contraste, otros asuntos de interés exclusivo para ciertos grupos quedan de lado y son atendidos de manera separada. Con todo, esta estrategia no está exenta de retos y tensiones. Después de todo, las organizaciones pueden tener distintas prioridades, pueden representar intereses incompatibles, estar relacionados estrechamente con grupos frente a los que otras organizaciones son hostiles - como profesionales del área de la salud y el cuidado o familiares de personas con discapacidad-o pueden tener que competir entre sí por recursos limitados (Sherlaw y Hudebine, 2015: 15).

\section{Hacia un proceso constituyente inclusivo para la ciudadanía}

Diversos estudios han puesto de relieve que preocuparse por la participación política de las personas con discapacidad es una cuestión que debe extenderse más allá de la consagración formal de derechos en los textos de derecho positivo: requiere explorar en qué medida los Estados elaboran planes y destinan recursos para asegurar que los espacios de participación política sean accesibles para las personas con discapacidad (Priestley y otros, 2016: 5-6; Schur, Kurse y Blanck, 2013: 107-109). En consecuencia, la participación política en el proceso constituyente exige la adopción de medidas de accesibilidad y ajustes razonables para personas con discapacidad. Tales medidas y ajustes deben verificarse en cuatro momentos diferenciados: antes, durante y después de cada proceso electoral o plebiscitario, y durante el funcionamiento del órgano constituyente. A las medidas que corresponden a esos cuatro momentos cabe agregar otras de carácter transversal, que deben proyectarse a aquéllos. 


\section{Antes del proceso electoral o plebiscitario}

Las asambleas, consultas u otras reuniones públicas que puedan llevarse a cabo en el marco del proceso constituyente deben hacerse en entornos físicamente accesibles y que cuenten con rampas y áreas de asientos especiales, por ejemplo. Cabe señalar que, para estos efectos, la accesibilidad física de los entornos incluye también el transporte, la educación, la información y las comunicaciones en el propio idioma, los nuevos sistemas y tecnologías, además de los sitios web de los órganos públicos y otros servicios e instalaciones abiertos o prestados al público, tanto en los entornos urbanos como en los rurales (Observación General 7, párrafo 45).

Además, es necesario que los insumos impresos o audiovisuales para la discusión estén disponibles en formatos accesibles (braille, subtítulos, fuentes tipográficas inclusivas, lengua de señas, textos en lectura fácil, lenguaje claro, pictogramas, sistemas de comunicación alternativos y aumentativos, etcétera) y que sean ampliamente difundidos entre las personas con discapacidad, las organizaciones, los partidos políticos y la sociedad civil en general. En este punto, debe aclararse que la información general entregada al público es muchas veces insuficiente para lograr incluir a las personas con discapacidad y el Estado debería preocuparse de hacer esa información accesible y cerciorarse que las personas con discapacidad la tengan a su disposición.

\section{Durante el proceso electoral o plebiscitario}

Según el artículo 29 de la CDPD, en los procesos electorales deben observarse tres aspectos para permitir la participación de personas con discapacidad. Primero, se debe garantizar que los procedimientos, instalaciones y materiales electorales sean adecuados, accesibles y fáciles de entender y utilizar. ${ }^{10}$ En segundo lugar, se debe proteger su derecho a emitir su voto en secreto en elecciones y referéndum públicos, sin intimidación. Por último, se debe garantizar su libre expresión de la voluntad como electores y, a este fin, cuando sea necesario y a petición de ellas, permitir que una persona de su elección les preste asistencia para votar.

En relación con la exigencia de accesibilidad, se ha advertido que su ausencia en los centros de votación, además de dificultar el ejercicio del voto para las personas con discapacidad, tiene un fuerte componente expresivo: envía el mensaje de que no se espera que ellas participen en la esfera política (Schur, Ameri y Adya, 2017: 1.375). En cualquier caso, debe tenerse presente que los remedios para afrontar las barreras asociadas a la falta de accesibilidad de los espacios de participación política varían

10. En este sentido, en su Observación General 2 (párrafo 43), el Comité ha sostenido que la obligación de proporcionar accesibilidad (artículo 9 de la CDPD) juega un rol fundamental para que las personas con discapacidad ejerzan su derecho a participar en la vida política y pública en igualdad de condiciones con las demás. 
de acuerdo con el tipo y a la intensidad de la discapacidad. En este sentido, estudios empíricos han mostrado que las medidas de accesibilidad impactan de manera dispar en la experiencia de ejercer el derecho de sufragio, según cuál sea el tipo de discapacidad de la persona (van Hees, Boije y de Putter, 2019: 819-836). Por ejemplo, la incorporación de rampas de acceso en los establecimientos electorales y disponer de casetas de votación más espaciosas puede generar un impacto favorable en la experiencia de votación de las personas con discapacidad física, pero no tener un impacto en la experiencia de personas con discapacidad intelectual. En este último caso, una estrategia de accesibilidad adecuada — por ejemplo— sería el diseño y elaboración de materiales electorales en formato de lectura fácil.

Fuera de estas exigencias, resulta pertinente orientar a los órganos electorales y a las organizaciones de personas con discapacidad para que capaciten a quienes se desempeñen como vocales o apoderadas de mesa (Rattray y Lako, 2018: 39). Una orientación de esta naturaleza podría ser de especial relevancia para el efectivo ejercicio del derecho a sufragio de las personas con discapacidad, tomando en cuenta que, en Chile, conforme el artículo 67 de la Ley sobre Votaciones Populares y Escrutinios, corresponde a la mesa respectiva determinar si corresponde otorgarles preferencia para emitir su voto, si procede otorgarles una ampliación del tiempo para votar o si la entidad de la discapacidad amerita la asistencia de otros para la emisión del voto.

\section{Después del proceso electoral o plebiscitario}

Una vez concluido cada proceso electoral, debe brindarse apoyo tanto a las personas con discapacidad como a las organizaciones para asegurar que la información sobre eventuales reclamaciones se encuentre disponible en formatos accesibles y que los espacios físicos o virtuales para formular tales reclamaciones lo estén también (Rattray y Lako, 2018: 39).

\section{Otras medidas transversales}

Para una adecuada implementación de todas estas medidas es indispensable contar, en forma previa, con información relativa a todas las personas con discapacidad en el país, así como con una evaluación de las barreras que enfrentan y de sus necesidades. El Comité sugiere un sistema estadístico unificado que permita recopilar datos de calidad, suficientes, oportunos y fiables (Observación General 7, párrafo 91). Dichos datos deben estar desglosados por sexo, edad, origen étnico, población rural o urbana, tipo de discapacidad y situación socioeconómica.

Asimismo, es crucial impulsar en forma sistemática campañas públicas de educación en torno a los derechos de personas con discapacidad, para promover su empoderamiento y crear conciencia de su posición de vulnerabilidad en la sociedad. 
El artículo 8 de la CDPD, relativo a la «toma de conciencia», ofrece una buena guía orientar la actividad estatal en este sentido. Al efecto, deben elaborarse programas sistemáticos de toma de conciencia, que comprendan campañas en los medios de comunicación a través de las emisoras de radio y los canales de televisión pública (párrafo 76). Dichos programas han de abarcarse toda la diversidad de personas con discapacidad como titulares de derechos y basarse en el modelo de derechos humanos de la discapacidad, a fin de erradicar estereotipos sociales arraigados.

\section{Hacia un órgano constituyente inclusivo}

\section{¿Cómo hacer inclusivo el órgano constituyente?}

Un proceso constituyente inclusivo no sólo debe preocuparse que las personas con discapacidad puedan participar a través de elecciones y plebiscitos. Un aspecto fundamental de la participación supone que ellas puedan integrar el órgano constituyente mismo. En este sentido, el artículo 29 del CDPD consagra el derecho de las personas con discapacidad a presentarse efectivamente como candidatas en procesos electorales, ejercer cargos y desempeñar cualquier función pública a todos los niveles de Gobierno, incluyendo la integración de un órgano constituyente. Si bien no es común que haya normas que prohíban la participación de personas con discapacidad en cargos de elección popular (Raub y otros, 2016: 233-235), hemos visto que ellas experimentan importantes barreras para acceder a las instancias de participación política. Como una forma de contrarrestar estas barreras y entendiendo que la exigencia de inclusión de las personas con discapacidad en la participación política debe expresarse en la adopción de medidas afirmativas, en la literatura comparada han sido considerados dos tipos de medidas.

Una primera alternativa corresponde al establecimiento de cuotas de participación de personas con discapacidad. Éstas pueden asumir la forma de escaños reservados, como ocurre en las elecciones de la Asamblea Nacional de Uganda, ${ }^{11}$ o de cuotas en las listas electorales, como sucede en las elecciones municipales de Túnez. ${ }^{12}$ Aunque esta estrategia puede contribuir a generar una minoría crítica de personas con discapacidad capaz de ejercer alguna influencia decisiva en los contenidos del nuevo texto constitucional, tiene el potencial inconveniente de generar el fenómeno del single-issue label: ${ }^{13}$ con esta terminología se designa a la práctica de asumir que so-

11. «Report of the United Nations Consultative Expert Group Meeting on international norms and standards relating to disability», United Nations Enable, disponible en https://bit.ly/3njqxb7.

12. "Political participation of women and men with disabilities in Tunisia: An IFES assessment», International Foundation for Electoral Systems, 27 de diciembre de 2018, disponible en https://bit. ly/2KoZFba.

13. «Guidelines on promoting the political participation of persons with disabilities», Organization for 
lamente los representantes con discapacidad abordarán cuestiones relacionadas con la discapacidad.

Respondiendo a dicho inconveniente, se ofrece una segunda alternativa, complementaria a la anterior, que consiste en disponer de consultas con personas con discapacidad, a través de las organizaciones de personas con discapacidad. Respecto de este punto, debe recordarse que, según lo expresado por el Comité, las consultas deben basarse en la transparencia, el respeto mutuo, el diálogo genuino y la voluntad sincera de alcanzar acuerdos colectivos y deben desarrollarse conforme a procedimientos que respondan a la diversidad de las personas con discapacidad (Observación General 7, párrafo 47). Por otro lado, «a fin de asegurarse de que no se deja a nadie atrás en relación con los procesos de consulta, los Estados parte deberían designar a personas encargadas de hacer un seguimiento de la asistencia, detectar grupos subrepresentados y velar por que se atiendan los requerimientos de accesibilidad y ajustes razonables» (párrafo 76 ).

\section{La reforma constitucional sobre cuotas para las personas con discapacidad}

Inmediatamente tras el acuerdo del 15 de noviembre de 2019, que estableció las bases para la creación de una nueva constitución para Chile, muchas voces se levantaron para demandar un órgano constituyente inclusivo. La reproducción de las estructuras de representación que hoy existen en el Congreso Nacional, tan deslegitimadas, no podían garantizar que la constitución expresara las demandas que habían movilizado a la ciudadanía. Tres ideas han monopolizado la discusión sobre la inclusividad: la paridad de género, los escaños reservados para los pueblos indígenas y la flexibilización de las reglas para la presentación de candidaturas independientes. Las prioridades de la asamblea constituyente inclusiva no consideraban a las personas con discapacidad. Esa situación, sin embargo, cambió sorpresivamente.

El 17 de diciembre del año 2019 ingresó a tramitación legislativa el proyecto de reforma constitucional mediante el cual se establecen reglas complementarias al acuerdo por una nueva constitución, dirigido a la conformación del órgano constituyente que elaborará una nueva constitución política (Boletín 13.129-07). A pesar de que el proyecto original sólo se limitaba a establecer una única disposición transitoria relativa a la reserva de escaños para los pueblos originarios, el desarrollo de la discusión parlamentaria en la Cámara de Diputados culminó en la adición de una nueva disposición, específicamente pensada para la participación de personas con discapacidad. ${ }^{14}$ Luego de una extensa discusión parlamentaria y transcurrido casi un año del

Security and Co-operation in Europe, 15 de marzo de 2019, disponible en https://bit.ly/37jJIMt.

14. La incorporación de esta disposición tuvo su origen en una indicación presentada por el diputado Miguel Mellado, de Renovación Nacional. 
inicio de su tramitación, el proyecto de reforma constitucional fue aprobado por el Congreso Nacional. ${ }^{15}$ En lo que concierne a la participación política de las personas con discapacidad, la reforma incorpora al actual texto constitucional la disposición transitoria cuadragésima séptima. Conforme a esta disposición, en las elecciones de los convencionales constituyentes, las listas electorales conformadas por un solo partido político y los pactos electorales de partidos políticos deberán reservar, a lo menos, el $5 \%$ del total respectivo de sus candidaturas para personas con discapacidad. ${ }^{16}$

La aprobación de esta reforma constitucional marca un hito en lo referente a la participación política de las personas con discapacidad. En primer lugar, porque refuerza la idea de que el punto de vista de las personas con discapacidad representa un insumo valioso para la elaboración del acuerdo fundamental de nuestra comunidad política, haciéndose parte del proceso de discusión y deliberación que se avecina. En segundo lugar, porque permite anticipar una mayor promoción de los derechos de las personas con discapacidad en el contexto de la discusión del nuevo texto constitucional. Como ya hemos mencionado, muchos de los derechos constitucionales tienen profundas consecuencias para las personas con discapacidad, lo que hace deseable que éstos sean estructurados de forma de tener en mente el disfrute de sus derechos y la satisfacción de sus necesidades.

\section{Cuestiones problemáticas}

Sin perjuicio de que la reforma constitucional aprobada pueda entenderse en términos positivos para la inclusión de las personas con discapacidad en el proceso constituyente, pueden advertirse ciertas cuestiones problemáticas que se proyectan tanto en la forma en que se desarrolló la discusión parlamentaria sobre el tema, como en la futura implementación de la reforma. Ahora bien, a pesar de estar ancladas en un comentario a la reforma constitucional recientemente aprobada, las observaciones que siguen resultan aplicables para cualquier iniciativa que tenga por objeto promover la participación política de las personas con discapacidad como miembros de órganos de representación. A su vez, estas observaciones pueden ser proyectadas a instancias de representación en las instituciones políticas que el órgano constituyente tiene encargo de diseñar.

15. Al cierre de la edición de este artículo (diciembre de 2020), el último hito registrado en la tramitación del proyecto, el 17 de diciembre de 2020, daba cuenta del oficio enviado al Ejecutivo comunicando la aprobación del proyecto de reforma constitucional por parte de Congreso Nacional.

16. Se establece, además, que el incumplimiento de esta exigencia $-\mathrm{y}$ la infracción a las reglas de acreditación de la discapacidad previstas- acarreará el rechazo de todas las candidaturas declaradas de los partidos o pactos electorales infractores. 


\section{Órgano constituyente accesible}

Tratándose de la elección de convencionales constituyentes, es necesario garantizar que las personas con discapacidad puedan efectivamente asumir y ejercer sus cargos. Para ello, resulta esencial que gocen de accesibilidad óptima a los procedimientos, información e instalaciones que correspondan (Rattray y Lako, 2018: 39). Al respecto, estudios que se han preocupado de documentar las experiencias de personas con discapacidad en el acceso o ejercicio de cargos públicos dan cuenta de que las barreras derivadas de la ausencia de accesibilidad se expresan en distintas instancias, como la participación en actos de campaña, la participación en la discusión y deliberación al interior del órgano colegiado en el que participan, la distribución de la información relevante para la toma de decisiones, etcétera (Evans y Reher, 2020; Waltz y Schippers, 2020).

De este modo, el establecimiento de cuotas en las listas electorales es sólo un paso inicial en el recorrido hacia a la inclusión de las personas con discapacidad en el órgano y en el debate constituyente. Este recorrido hacia la inclusión requerirá de la elaboración de una estrategia de accesibilidad que, en primer lugar, sea sensible a la diversidad de las personas con discapacidad —en el sentido de prever distintas medidas de accesibilidad de acuerdo con el tipo de discapacidad-; y que, en segundo lugar, sea comprensiva de las distintas instancias o momentos de participación en el seno de la discusión constituyente, según se expuso más arriba. Esta estrategia posibilitará que, de resultar electas personas con discapacidad para servir como convencionales constituyentes, se puedan proveer las medidas de adecuación que se requieran para su participación en igualdad de condiciones, las que podrían incluir, por ejemplo, ramplas de acceso, asientos adaptados o preferenciales, lectoescritura braille, traducción en lengua de señas, distribución de información escrita en formato de lectura fácil, modalidades de asistencia personal, etcétera.

\section{Heterogeneidad de las personas con discapacidad como grupo social}

Una segunda cuestión que considerar, ya sugerida más arriba, es la diversidad en tanto grupo social. Las personas con discapacidad son un grupo heterogéneo, integrado por personas con tipos de discapacidad distintos - por ejemplo, discapacidades físicas, sensoriales, intelectuales y psicosociales- $-{ }^{17}$ En este escenario de diversidad al interior de las personas con discapacidad se aloja un fenómeno de especial impacto

17. En el derecho chileno, la Ley 20.422 y el Decreto 47/2012 del Ministerio de Salud, que aprueba el Reglamento para la calificación y certificación de la discapacidad, no se refieren a tipos de discapacidades sino a tipos de deficiencias. Las categorías utilizadas por estos textos de derecho positivo son: deficiencia física, deficiencia sensorial, deficiencia mental de causa síquica y deficiencia mental de causa intelectual. 
para el cumplimiento de las cuotas electorales que establece la reforma: el problema de la «jerarquía de las deficiencias» (hierarchy of impairments) (Deal, 2003: 897-910; Thomas, 2000: 1.155-1.156; Tringo, 1970: 295-306). Esta expresión se ha utilizado en los estudios sobre discapacidad para transmitir la idea de que las percepciones y actitudes hacia las personas con discapacidad varían y usualmente dependen del tipo y el origen de las deficiencias que ellas tienen. Algunos han abordado este problema en relación con la designación o elección en cargos públicos, indicando que existen diferencias en las percepciones del público con respecto a una persona con discapacidad según se trate del origen de la deficiencia, esto es, sobrevenida o de nacimiento, o se trate de una discapacidad física-sensorial o intelectual-mental, esto es, según el tipo de deficiencia (Waltz y Schippers, 2020: 9-10). Comparativamente, se sugiere que es más probable que las personas con una discapacidad originada al nacer y las personas con discapacidad intelectual-mental sean destinatarias de percepciones adversas o desfavorables.

Teniendo en cuenta lo anterior, el hecho de que la reforma constitucional establezca cuotas electorales bajo una fórmula genérica como la de «personas en situación de discapacidad» deja abierta la posibilidad de que opere la «jerarquía de las deficiencias» y que, en consecuencia, se prefiera la selección de candidatos y candidatas con ciertos tipos de discapacidades por sobre otras. Al respecto, un estudio sobre la presentación de personas con discapacidad como candidatos en las elecciones provinciales del año 2013 en Columbia Británica (Canadá) detectó que todas las personas que se presentaron como candidatos a las elecciones tenían discapacidad física (usuarios de sillas de ruedas) y que, por lo tanto, sus experiencias no reflejaban las experiencias de las personas con otros tipos de discapacidades (Langford y Levesque, 2017: 11).

Que la reforma constitucional no sea sensible a la heterogeneidad y diversidad dentro de las personas con discapacidad constituye un obstáculo de particular relevancia para que, mediante su participación, el proceso constituyente pueda enriquecerse con sus experiencias y para que sus distintos intereses, demandas y perspectivas puedan ser consideradas debidamente. Tomarse en serio la inclusión de las personas con discapacidad en el proceso constituyente debiese significar ser conscientes de dicha diversidad y, en consecuencia, favorecer que las distintas realidades y experiencias que integran la discapacidad se vean representadas.

\section{Certificación de la discapacidad}

Una tercera cuestión para tener en cuenta en la implementación de la reforma constitucional dice relación con la certificación de la situación de discapacidad de quienes funjan como candidato o candidata a la convención: ¿con base en qué criterio se evaluará el cumplimiento la cuota? A primera vista, esto puede parecer una cuestión meramente técnica, pero encierra problemas de representación complejos. 
A diferencia de lo que sucede con los problemas de accesibilidad y de la jerarquía de las deficiencias, la cuestión de cómo determinar la situación de discapacidad sí fue advertida y tratada durante la tramitación legislativa de la reforma. Específicamente en la discusión del proyecto en el Senado, este punto fue abordado por el representante del Servicio Electoral, ${ }^{18}$ por el Subsecretario de Servicios Sociales ${ }^{19}$ y por distintas indicaciones sustitutivas presentadas por senadores y senadoras. ${ }^{20}$

El debate parlamentario denota que la pregunta sobre cómo determinar el «estatus» de discapacidad de la persona que se presenta como candidato o candidata no es trivial. Esto es así porque la categoría de "persona con discapacidad» es una de contornos difusos y que, además, se encuentra en constante evolución (Beaudry, 2020: 3-21). Incluso, se ha sostenido que el concepto de discapacidad tiene la calidad de concepto esencialmente controvertido (Silvers, 2020: 22-45) y, en consecuencia, no siempre resulta tan claro determinar si un individuo es o no una persona con discapacidad.

Como consecuencia de la discusión parlamentaria, el texto aprobado de la reforma constitucional aborda expresamente la cuestión de la acreditación de la discapacidad del candidato, admitiendo dos vías alternativas. Por una parte, el candidato o la candidata puede acreditar su situación de discapacidad por medio de los procedimientos de calificación y certificación regulados en la Ley 20.422, sobre Igualdad de Oportunidades e Inclusión Social de las Personas con Discapacidad. ${ }^{21}$ Por otra parte, el candidato o la candidata también puede acreditar su situación de discapacidad demostrando ser asignatario de una pensión de invalidez de cualquier régimen previsional.

Un factor adverso que debe considerarse a la hora de recurrir a formas de acreditación «institucional» de la discapacidad es que los procedimientos de calificación y certificación sólo operan a petición de parte interesada, conforme al artículo 13 de la

18. El representante del Servicio Electoral indicó expresamente que surgían dudas en torno a cómo se iba a acreditar la situación de discapacidad para los efectos del cumplimiento de la cuota. Boletín 13.129-07, Informe de la Comisión de Constitución, Legislación, Justicia y Reglamento del Senado, p. 8.

19. Expresando la posición del Gobierno, el subsecretario indicó que una vía para acreditar la situación de discapacidad del candidato consistía en requerir la calificación y certificación de la discapacidad prevista en la Ley 20.422. No obstante, añadió que la discapacidad también podría acreditarse mediante la calidad de asignatario de pensión de invalidez en cualquier régimen previsional. Boletín 13.129-07, Segundo Informe de la Comisión de Constitución, Legislación, Justicia y Reglamento del Senado, p. 100. 20. Véase Boletín 13.129-07, Informe de la Comisión de Constitución, Legislación, Justicia y Reglamento del Senado, p. 8, y Segundo Informe de la Comisión de Constitución, Legislación, Justicia y Reglamento del Senado, pp. 107-110.

21. Esta fórmula, valga recordar, es idéntica a la que quedó consagrada en el artículo 157 bis del Código del Trabajo y que es utilizada para los efectos de la cuota de empleo para personas con discapacidad establecida por la Ley 21.015, que Incentiva la Inclusión de Personas con Discapacidad al Mundo Laboral. 
Ley 20.422. Lo mismo ocurre con la asignación de pensiones de invalidez, conforme al artículo 65 bis del Decreto Ley 3.500 y al artículo 18 de la Ley 20.255. Ello sugiere que no toda persona con discapacidad en Chile cuenta con la respectiva certificación de la COMPIN, ni es asignataria de pensión de invalidez. Además, según el artículo 4 de la Ley 20.422, los procedimientos de calificación y certificación de la discapacidad están destinados al acceso a los beneficios y prestaciones sociales contemplados en dicha ley; no tienen un «efecto constitutivo» de la situación de discapacidad. Sumado a ello, debe considerarse que la definición de «persona con discapacidad» establecida en el artículo 5 de la Ley 20.422 no vincula la situación de discapacidad con los procedimientos de calificación y certificación, ni mucho menos con la calidad de asignatario de pensión de invalidez.

Debido a lo anterior, una vía alternativa o complementaria a la identificación mediante estas formas de acreditación institucional hubiese sido la demostración de la afiliación a una organización de personas con discapacidad. Las organizaciones operan como plataformas significativas para la promoción y el ejercicio de los derechos de las personas con discapacidad, proveyéndoles de una voz para expresar sus puntos de vista, necesidades y prioridades (Young, Reeve y Grills, 2016: 45-71). La experiencia de participar en una organización de personas con discapacidad tiene impactos positivos para su participación en los procesos políticos. En este sentido, entrevistas a personas con discapacidad en cargos políticos formales evidencian dos factores comunes: en primer lugar, previo al ejercicio de sus cargos políticos, todos los entrevistados habían participado como miembros de organización o de grupos de defensa de los derechos de las personas con discapacidad; en segundo lugar, todos los entrevistados sostuvieron que tal experiencia previa les había proporcionado habilidades útiles para desenvolverse posteriormente en el campo de la «política formal» (Langford y Levesque, 2017: 11). Si esto es así, la selección de candidatos que cuenten con una afiliación a organización constituiría una forma de potenciar la voz de las personas con discapacidad en el debate constituyente.

\section{Discusión sin participación}

Además de las interrogantes en torno a la futura implementación de la reforma, puede añadirse una segunda clase de preocupación, asociada a cómo se sustanció la discusión parlamentaria de la reforma constitucional aprobada. En este sentido, resulta preocupante observar la escasa participación que tuvieron las personas con discapacidad durante la tramitación de la reforma. Las actas e informes, tanto de la Cámara de Diputados como del Senado, dan cuenta de que las organizaciones de personas con discapacidad no fueron invitadas a expresar su punto de vista respecto de una reforma constitucional que las involucra directamente. La voz y perspectiva de las personas con discapacidad sólo se vio representada en la participación de María 
Soledad Cisternas, quien fue invitada en su calidad de enviada especial sobre Discapacidad y Accesibilidad del Secretario General de las Naciones Unidas. ${ }^{22} \mathrm{La}$ ausencia de las personas con discapacidad y sus organizaciones representativas se hace más llamativa si se contrasta con la alta participación y convocatoria de los representantes de los pueblos originarios.

Ahora bien, más allá de que esta situación sea claramente contraria a lo dispuesto en el artículo 4 párrafo 3 de la CDPD, la participación de personas con discapacidad en los procesos de discusión y adopción de políticas públicas tiene un impacto sustantivo en orden a que dichas políticas sean sensibles a la situación de las personas con discapacidad y a los problemas que ellas experimentan (Langford y Levesque, 2017: 14). En este sentido, en otras jurisdicciones, diversas iniciativas de consulta y acción conjunta con personas con discapacidad y organizaciones han resultado exitosas para identificar, monitorear y corregir los problemas de accesibilidad asociados a la participación electoral de las personas con discapacidad (Schur, Ameri y Adya, 2017: 1.387). Ello sugiere que haber contado con la presencia y participación activa de las personas con discapacidad en la discusión del proyecto hubiese contribuido en gran medida a abordar los problemas de implementación que hemos identificado.

\section{Conclusiones}

La vida de las personas con discapacidad es determinada no sólo por su contingente posición social, sino también por su mayor dificultad para satisfacer sus necesidades, ejercer sus derechos y lograr reconocimiento de sus diferencias. Una transformación del modelo de sociedad que implique una inyección de igualdad y solidaridad podría impactar positivamente la vida de las personas con discapacidad. Esa transformación social podría ser impulsada por el cambio constitucional. Sin embargo, ello enfrenta dos tipos de desafíos. En primer lugar, requiere un proceso constituyente que habilite a la democracia para llevar a cabo las transformaciones que permitan satisfacer las demandas sociales de la ciudadanía, lo que no está de ninguna manera asegurado. En segundo lugar, esta vez en relación con las personas con discapacidad, requiere que sus intereses, junto con sus derechos y perspectivas, sean considerados en el proceso constituyente, lo que, como se ha visto, tampoco está garantizado.

El derecho internacional de los derechos humanos ha desarrollado el derecho de participación de las personas con discapacidad, por un lado, en relación con su derecho a no ser excluidos del padrón electoral y tener acceso a sufragar, y, por otro lado, en relación con su derecho de participar políticamente a través de sus propias asociaciones. Estas últimas son el principal medio mediante el cual los Estados pueden dar

22. Boletín 13.129-07, Segundo Informe de la Comisión de Constitución, Legislación, Justicia y Reglamento del Senado, pp.51-55. 
cumplimiento a su deber de consultar a las personas con discapacidad en los asuntos que se relacionen con ellas. Además, la participación política en el proceso constituyente exige la adopción de medidas de accesibilidad y ajustes razonables. Estas medidas y ajustes deben adoptarse antes, durante y después de cada proceso electoral o plebiscitario, así como durante el funcionamiento del órgano constituyente.

Sin perjuicio de lo anterior, para que un proceso constituyente pueda calificarse como inclusivo, no basta con que las personas con discapacidad puedan participar en sus respectivos procesos electorales y plebiscitarios. Un aspecto fundamental de la participación supone que puedan también integrar el órgano constituyente mismo. En el marco de la discusión constituyente, la consideración de este aspecto se ha materializado en la reciente aprobación de una reforma constitucional que dispone que las listas electorales conformadas por un solo partido y los pactos electorales de partidos políticos que se presenten para la elección de los convencionales constituyentes estén conformadas, a lo menos en $5 \%$ de sus candidatos, por personas con discapacidad. Aunque la reforma constitucional apunta en la dirección correcta, en tanto busca la efectiva inclusión en el órgano constituyente, existen al menos dos aspectos de preocupación y que deben tomarse en cuenta. En primer lugar, una preocupación en la implementación: por una parte, se ha resaltado que la elaboración de una estrategia general de accesibilidad resulta esencial de cara a la futura puesta en práctica de la reforma; por otra parte, sin perjuicio de que el texto de la reforma constitucional establece criterios para determinar la situación de discapacidad del candidato a efectos del cumplimiento de la cuota, no se hace cargo de la heterogeneidad dentro de las personas con discapacidad. En segundo lugar, una preocupación en la gestación de la reforma: el punto de vista de las personas con discapacidad no puede estar ausente de la discusión. Una iniciativa que involucra directamente la situación de las personas con discapacidad exige su participación activa en el debate.

\section{Reconocimiento}

Este trabajo es el resultado del proyecto «El ejercicio de derechos fundamentales por las personas con discapacidad mental en Chile desde la perspectiva de los derechos humanos», proyecto Fondecyt 1190343, ANID-Chile, del cual Pablo Marshall es investigador principal y Eduardo Marchant es tesista.

\section{Referencias}

BEAUdRY, Jonas-Sébastien (2020). «Theoretical strategies to define disability». En Adam Cureton y David Wasserman (editores), The Oxford handbook of philosophy and disability (pp. 3-21). Nueva York: Oxford University Press. 
BIRTHA, Magdolna (2013). «Nothing about CRPD monitoring without us: A case study on the involvement of the disability movement in policy-making in Zambia». African Disability Rights Yearbook, 1:115-138. Disponible en https://bit.ly/3gKBHDj.

Bundy, Hollister (2003). «Election reform, polling place accessibility, and the voting rights of the disabled». Election Law Journal: Rules, Politics, and Policy, 2 (2): 217240. DOI: 10.1089/153312903321578197.

DEAL, Mark (2003). «Disabled people's attitudes toward other impairment groups: A hierarchy of impairments». Disabilty \& Society, 18 (7): 897-910. DOI: 10.1080/0968759032000127317.

Degener, Theresia (2017). "A new human rights model of disability». En Valentina della Fina, Rachele Cera y Giuseppe Palmisano (editores), The United Nations Convention on the Rights of Persons with Disabilities: A commentary (pp. 41-59). Cham: Springer International Publishing. DOI: 10.1007/978-3-319-43790-3_2.

Evans, Elizabeth y Stephanie Reher (2020). «Disability and political representation: Analyzing the obstacles to elected office in the UK». International Political Science Review, 0: 1-16. DOI: 10.1177/0192512120947458.

Goodley, Dan (2014). Dis/ability studies: Theorising disablism and ableism. Nueva York: Routledge.

Herring, Jonathan (2013). Caring and the law. Oxford: Hart.

Kamundia, Elizabeth (2014). «Kenya». African Disability Rights Yearbook, 2: 185-205. Disponible en https://bit.ly/34c5JdQ.

LANG, Raymond, Maria Kett, Nora Groce y Jean-Francois Trani (2011). «Implementing the United Nations Convention on the rights of persons with disabilities: Principles, implications, practice and limitations». ALTER, European Journal of Disability Research, 5: 206-220. DOI: 10.1016/j.alter.2011.02.004.

LANGFORD, Brynne y Mario Levesque (2017). «Symbolic and substantive relevance of politicians with disabilities: A British Columbia case study». Canadian Parliamentary Review, 40 (2): 8-17. Disponible en https://bit.ly/2IRgoU4.

Levesque, Mario y Brynne Langford (2016). «The role of disability groups in the development and implementation of the UN Convention of the Rights of Persons with Disabilities». Canadian Journal of Disability Studies, 5 (4): 62-102. DOI: 10.15353/cjds.v5i4.315.

Löve, Laufey, Rannveig Traustadóttir y James Rice (2019). «Shifting the balance of power: The strategic use of the CRPD by disabled people's organizations in securing "a seat at the table"». Laws, 8 (11): 1-15. DOI: 10.3390/laws8020011.

Marshall, Pablo y Patricia Abollado (2019). «El sufragio de las personas con discapacidad intelectual en Chile». En Nicolás Espejo y Fabiola Lathrop (editores), Derecho y discapacidad Intelectual. Santiago: Thomson Reuters.

Priestley, Mark, Martha Stickings, Ema Loja, Stefanos Grammenos, Anna Lawson, Lisa Waddington y Bjarney Fridriksdottir (2016). «The political participation of 
disabled people in Europe: Rights, accessibility and activism». Electoral Studies, 42: 1-9. DOI: 10.1016/j.electstud.2016.01.009.

RatTraY, Sarah y Entela Lako (2018). Disability inclusive development in UNDP: Guidance and entry points. Nueva York: UNDP.

Raub, Amy, Isabel Latz, Aleta Sprague y Michael Ashley Stein (2016). "Constitutional rights of persons with disabilities: An analysis of 193 National Constitutions». Harvard Human Rights Journal, 29: 203-240.

SCHur, Lisa, Mason Ameri y Meera Adya (2017). «Disability, voter turnout, and polling place accessibility». Social Science Quarterly, 98 (5): 1.374-1.390. DOI: 10.1111/ ssqu.12373.

SCHUr, Lisa, Douglas Kurse y Peter Blanck (2013). People with disabilities: Sidelined or mainstreamed?. Nueva York: Cambridge University Press.

Sherlaw, William y Hervé Hudebine (2015). «The United Nations Convention on the Rights of Persons with Disabilities: Opportunities and tensions within the social inclusion and participation of persons with disabilities». ALTER, European Journal of Disability Research, 9: 9-21. DOI: 10.1016/j.alter.2014.08.001.

Silvers, Anita (2020). «In pursuit of justice for disability: Model neutrality revisited». En Adam Cureton y David Wasserman (editores), The Oxford handbook of philosophy and disability (pp. 22-45). Nueva York: Oxford University Press.

Thomas, Adrian (2000). «Stability of Tringo's hierarchy of preference toward disability groups: 30 years later». Psychological Reports, 86 (3): 1.155-1.156. DOI: 10.2466/ pro.2000.86.3C.1155.

Toritsyn, Arkadi y A.H. Monjurul Kabir (2013). Promoting the human rights of persons with disabilities in Europe and the Commonwealth of independent States: Guide. Nueva York: UNDP.

Tremain, Shelley (2016). «Knowing disability, differently». En Iann James Kidd, José Medina y Gaile Pohlhaus (editores), The Routledge Handbook on Epistemic Injustice (pp. 175-183). Nueva York: Routledge. DOI: 10.4324/9781315212043.

Tringo, John (1970). «The hierarchy of preference toward disability groups». The Journal of Special Education, 4 (3): 295-306. DOI: 10.1177/002246697000400306.

Van Hees, Suzanne, Hennie Boije e Iris de Putter (2019). «Voting barriers and solutions: The experiences of people with disabilities during the Dutch national election in 2017». Disability \& Society, 34 (5): 819-836. DOI: 10.1080/09687599.2019.1566052.

Waldschmit, Anne, Andreas Sturm, Anemari Karačić y Timo Dins (2017). «Implementing the UN CRPD in European countries: A comparative study on the involvement of organisations representing persons with disabilities». En Rune Halvorsen, Bjørn Hvinden, Jerome Bickenbach, Delia Ferri y Ana Marta Guillén Rodríguez (editores), The changing disability policy system: Active citizenship and disability in Europe (pp. 177-195). Nueva York: Routledge. DOI: 10.4324/9781315623931-11. 
WALTZ, Mitzi y Alice Schippers (2020). «Politically disabled: Barriers and facilitating factors affecting people with disabilities in political life within the European Union». Disability \& Society, 35 (8): 1.290-1.314. DOI: 10.1080/09687599.2020.1751075.

Wolbring, Gregor (2008). «The politics of ableism». Development, 51 (2): 252-258. DOI: $10.1057 /$ dev.2008.17.

Young, Rebekah, Matthew Reeve y Nathan Grills (2016). «The functions of disabled people's organisations (DPOs) in low and middle-income countries: A literature review». Disability, CBR \& Inclusive Development, 27 (3): 45-71. DOI: 10.5463/dcid. v27i3.539.

\section{Sobre los autores}

Pablo Marshall es abogado. Doctor en Derecho por la Universidad de Glasgow, Reino Unido. Licenciado en Ciencias Jurídicas y Sociales por la Universidad de Chile. Profesor de la Facultad de Derecho Ciencias Jurídicas en la Universidad Austral, Chile. Su correo electrónico es pmarshall@uach.cl. (D) https://orcid. org/0000-0001-8347-4620.

Viviana Ponce de León es abogada. Doctora en Derecho por la Pontificia Universidad Católica de Valparaíso, Chile. Licenciada en Ciencias Jurídicas y Sociales por la Universidad Católica del Norte, Chile. Profesora de la Facultad de Derecho Ciencias Jurídicas en la Universidad Austral, Chile. Su correo electrónico es viviana. poncedeleon@uach.cl. (D) https://orcid.org/o000-0001-7574-1717.

Eduardo Marchant es abogado. Licenciado en Ciencias Jurídicas y Sociales por la Universidad Austral, Chile. Estudiante de Doctorado en Derecho, Facultad de Derecho Ciencias Jurídicas en la Universidad Austral, Chile. Su correo electrónico es ecmarchantvivanco@gmail.com. (D) https://orcid.org/oooo-0002-1659-1357. 
El Anuario de Derechos Humanos es una publicación semestral de referencia y consulta en materia de derechos humanos y campos afines. Busca ser un espacio de discusión de los temas centrales en el ámbito nacional e internacional sobre derechos humanos. Es publicado desde 2005 por el Centro de Derechos Humanos de la Facultad de Derecho de la Universidad de Chile.

\author{
EDITORA \\ Claudia Iriarte Rivas \\ ciriarter@derecho.uchile.cl \\ SITIO WEB \\ anuariocdh.uchile.cl \\ CORREO ELECTRÓNICO \\ anuario-cdh@derecho.uchile.cl \\ LICENCIA DE ESTE ARTÍCULO \\ Creative Commons Atribución Compartir Igual 4.o Internacional
}

\author{
\% \\ La edición de textos, el diseño editorial \\ y la conversión a formatos electrónicos de este artículo \\ estuvieron a cargo de Tipográfica \\ (www.tipografica.io)
}

